\title{
Corrigendum to "Observations of a phytoplankton spring bloom onset triggered by a density front in NW Mediterranean" published in Ocean Sci., 10, 657-666, 2014
}

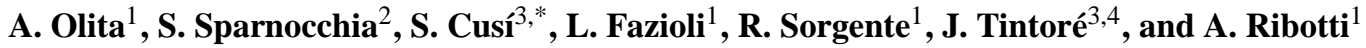 \\ ${ }^{1}$ CNR-IAMC, Institute for Coastal Marine Environment, Oristano Section, Torregrande (OR), Italy \\ ${ }^{2}$ CNR-ISMAR, Institute of Marine Sciences, Trieste Section, Trieste, Italy \\ ${ }^{3}$ SOCIB, Balearic Islands Coastal Observing and Forecasting System, Palma de Mallorca, Spain \\ ${ }^{4}$ IMEDEA (CSIC-UiB), Esporles, Spain \\ *now at: CSIC-UTM, Marine Technology Unit, Barcelona, Spain
}

Correspondence to: A. Olita (antonio.olita@cnr.it)

Due to an oversight in the production process, the article "Observations of a phytoplankton spring bloom onset triggered by a density front in NW Mediterranean" was published with a mistake in the affiliations. The first affiliation should be CNR-IAMC, Institute for Coastal Marine Environment, Oristano Section, Torregrande (OR), Italy (not SOCIB, Balearic Islands Coastal Observing and Forecasting System, Palma de Mallorca, Spain). 